Article

\title{
Longitudinal Effects of Violent Media Usage on Aggressive Behavior-The Significance of Empathy
}

\author{
Thomas Mößle *, Sören Kliem and Florian Rehbein \\ Criminological Research Institute of Lower Saxony (Kriminologisches Forschungsinstitut \\ Niedersachsen e.V.), Lützerodestraße 9, Hannover 30161, Germany; \\ E-Mails: soeren.kliem@kfn.de (S.K.); florian.rehbein@kfn.de (F.R.) \\ * Author to whom correspondence should be addressed; E-Mail: thomas.moessle@kfn.de; \\ Tel.: +49-(0)511-34836-75; Fax: +49-(0)511-34836-10.
}

Received: 12 September 2013; in revised form: 12 February 2014 / Accepted: 13 February 2014 / Published: 26 February 2014

\begin{abstract}
The aim of this study was to thoroughly investigate the link between violent media consumption and aggressive behavior. Using a large longitudinal student sample, the role of empathy as a possible mediator of this relationship was of special interest. Data were drawn from wave three to five of the Berlin Longitudinal Study Media, a four-year longitudinal control group study with 1207 school children. Participants completed measures of media usage (violent content of TV and computer games), aggressive behavior perpetration, and empathy. The average age of participants was 10.4 years at Time 1 and 12.4 years at Time 3 . Half of the study sample was male $(50 \%)$. Trivariate structural equation modeling using three measurement times were conducted for assessing the role of empathy as a mediator of the longitudinal relationship between the usage of violent media content and aggressive behavior. For male students empathic skills were shown to unfold a key mediating role between problematic media usage and aggressive behavior.
\end{abstract}

Keywords: media violence; aggressive behavior; empathy; longitudinal study; mediation analysis

\section{Introduction}

It is well known that television programs as well as video games often contain violent content [1-3]. With the increasing spread of the Internet and digital media in the past decade, a shift from television 
to the use of interactive media, as in video-sharing websites and video games could be observed [4]. Research further indicates that violent content, although in most countries only suitable for adult audiences, is also used by minors to a large extent [4-6]. With the presence of violent content on the Internet, access to this content by minors is even less restricted.

Regarding violent media effects, there is an ongoing controversial public and scientific debate on the (causal) effects of violent media consumption on aggression. On the one hand, several meta-analytic reviews could systematically show - for different age groups - that there is a positive association between the consumption of violent media content and various constructs of aggression, such as aggressive behavior, aggressive cognition, aggressive affect, violent behavior, desensitization, lack of empathy and lack of pro-social behavior [7-11]. Observed associations were shown to be in the small to medium range ( $r=0.15$ to $r=0.31$, Cohen's $d=0.30$ to Cohen's $d=0.65$; (e.g., [7-9]; for an overview see [12])), with the weakest effects for explicitly violent behavior. On the other hand, there are other meta-analytic reviews that question the reliability of these results due to publication bias and conclude that there is little support that media violence is associated with higher aggression [13-16]. Mean observed associations were shown to be in a small range $(r=0.08)$ in these reviews (e.g., [15]).

One reason for these diverging meta-analytic results might be the fact that media violence is by far not the only risk factor for aggression: For example, experienced personal victimization (cf. [17,18]), delinquent peers (cf. [19,20]), trait aggression (cf. [21,22]), empathy (cf. [23,24]), impulsivity (cf. [25,26]), gender (cf. [18,27]), school environment (cf. [28]) as well as parenting style and parental media usage (cf. [29]) are all supposed to be of interest as well. The more so, as most of them interact with a specific media usage behavior and possibly moderate (for trait aggression, cf. [28,30]) or mediate (for empathy or trait hostility, cf. [23]) the negative effect of violent media consumption on aggression.

In addition, there is still a scarcity of more recent longitudinal studies (starting in 2000) with a child or adolescent population that would truly allow untangling the causality of the relationship between violent media content consumption and constructs of aggression. Especially those studies with study intervals longer than one year and more than two measurements are very rare (2 measurements: $\leq 1$ year: [28,31-36], games only [37]; 2 years: [38], games only [39,40]; >5 years: [41], TV only [42-45]; $>2$ measurements: $\leq 1$ year: [46], games only [47]; 2 years: [17,48]; >5 years: TV only [49]). Furthermore, those longitudinal studies which have been conducted are also diverse in their findings: Some show long-term causal effects of repeated violent media exposure on aggression (e.g., [47]); others report the opposite, i.e., a selection effect (e.g., [36]). With regard to longitudinal studies with German child or adolescent samples (all four with two measurements), three studies provide cross-lagged evidence for a causal effect of violent media consumption on aggression [28,38,40], and one provides cross-lagged evidence for a selection effect [36]. Hopf and colleagues [38] could show with $N=314$ fifth to seventh graders that media violence at time 1 was a significant predictor of violent behavior at school at time 2 (two years later). In two studies by Möller and Krahé [40] with $N=143$ and Krahé and Möller [28] with $N=1,237$ seventh and eighth graders, video game violence exposure at time 1 was linked to physical aggression at time 2 (30 months later, 12 months later), whereas the opposite paths were non-significant. In a study by von Salisch and colleagues [36] with $N=324$ third and fourth graders, however, children who were rated as openly aggressive at time 1 showed a higher preference for violent computer games at time 2 (12 months later), whereas the opposite path from violent computer game preference to aggression, indicating a causal effect, was non-significant. 
An integrative framework for explaining short- and long-term as well as cognitive, emotional and behavioral effects of violent media content consumption is offered by the General Aggression Model $(G A M)$ by Anderson and colleagues [10,11,50], which combines several earlier explanatory models of aggression [51-56]. According to the $G A M$, as a short-term effect of violent media usage there is a higher probability of violent behavior caused by a change in appraisal and decision processes due to an aggression-like state with aggressive cognitions, aggressive affect and arousal during violent media usage. Long-term effects, caused by processes of learning, rehearsal and reinforcement of aggression-related knowledge structures in repeated reception phases of violent media content over a longer period of time, are: aggressive beliefs and attitudes, aggressive perceptual schemata, a hostile attribution bias, aggressive behavior scripts, and desensitization to fictional and real-life violence.

Regarding possible negative effects of violent media usage, various studies also focused on just the latter processes of desensitization and reduced empathic behavior (especially in real-life violence situations). According to the GAM [10,11,50], both outcomes can be considered as long-term effects of violent media exposure (cf. [7,28]), although especially in the case of desensitization, small short-term effects might also be observable [7]. Desensitization to violence and changes in empathic skills can be understood as a reduction of physiological and emotional responses to new fictional and real-life scenes of violence as well as a lower empathic reaction to victims of violence, which in turn reduces the level of inhibition of aggressive behavior [7,57]. Based on this understanding, aggression and violence are increasingly regarded as legitimate and as an effective way of attaining one's ends [58].

The assumption of reduced emotional responses to real life violence due to media violence in the sense of desensitization is supported by various experimental research results. In a quasi-experimental study with $N=39$ participants by Bartholow and colleagues [59], for example, habitual users of media violence showed decreased emotional reactions to pictures of real-life violence, even after controlling for trait aggression. In an experimental study with $N=257$ participants by Carnegey and colleagues [60], 20 min of violent video game use caused lower emotional reactions as indicated by a lower heart rate and a lower galvanic skin response in confrontation with filmed real-life violence. More recently, Krahé and colleagues [61] found effects of physiological desensitization in habitual media violence users compared with participants with a lower violent media usage in a quasi-experimental study with $N=303$ participants.

Evidence for the effect of violent media usage on reduced empathy, however, could primarily be drawn from cross-sectional and longitudinal studies. In a cross-sectional study with $N=150$ elementary school children, Funk and colleagues [62], for example, found that children with a higher violent video game consumption showed reduced empathy. Bartholow and colleagues [23] report in their cross-sectional study with $N=200$ male undergraduates (study 1) a significant link between violent video game exposure and lower empathy. In addition, empathy was shown to partially mediate the effect of violent video game exposure on aggression. Both effects, i.e., causal and mediating effect, could also be replicated in an experimental study using a subsample of $N=92$ participants (study 2). In the above mentioned short-term longitudinal study by Krahé and Möller [28], media violence at time 1 was also linked to lower empathy at time 2, whereas the opposite path from empathy at time 1 to media violence usage at time 2 was non-significant. Unfortunately, as the authors note, due to only two measurements being available, the role of empathy as a mediator of the relationship between media 
violence and aggression could not be tested properly. This restriction, in fact, applies to all other longitudinal studies with only two measurements (see above) testing any mediating effect.

In the current paper, we want to address this research gap. The link between violent media consumption and aggressive behavior is thoroughly investigated in a large population of children and adolescents using a longitudinal design with three measurements. We focus on the longitudinal effects of media violence on self-reported real-life aggressive behavior. Having three measurements, we want to explicitly focus on the role of empathy as a possible mediator of this relationship. The following research questions are to be answered:

(1) Is there a significant longitudinal relationship between violent media consumption and aggressive behavior?

(2) Is the relationship between violent media consumption and aggressive behavior significantly mediated by empathy?

\section{Method}

\subsection{Sample}

The Berlin Longitudinal Study Media is a four-year longitudinal control group study with 1207 school children (3rd-6th grade) representative of the (federal) state of Berlin. After study approval by the state school authorities (Berliner Senatsverwaltung für Bildung, Jugend und Sport), all classes with $n>15$ were selected for sampling (24,352 children in 1009 classes) from the overall population of 1042 Berlin third grade classes (status May 2005: 24,714 children). Eighty classes from different schools were drawn randomly, 47 of which participated in the longitudinal study (1129 children). A total of 943 children participated at t1 (84\% participation), 846 at t2 (88\%), 835 at t3 (87\%), 827 at t4 (83\%) and a total of 806 at t5 (79\%; for further details see $[5,12,63])$. Twenty primary school classes with 554 children were randomly assigned to school-based media lessons (intervention group; for details on the intervention see $[5,12,63,64])$. Twenty further classes with 512 children served as a control group. The remaining seven classes served as baseline classes (only $\mathrm{t} 1, \mathrm{t} 4, \mathrm{t} 5$ ).

Only children from the intervention and control classes, for whom data were available at $\mathrm{t} 3$, $\mathrm{t} 4$ or $\mathrm{t} 5$ and at least for two measurement occasions, were included in the sample, thus resulting in a total $n=724$. All baseline classes ( $n=7 ; 141$ students) were excluded from the analyses due to missing data at $\mathrm{t} 3$. Analyses were restricted to $\mathrm{t} 3 \mathrm{-t} 5$, as aggressive behavior was assessed at $\mathrm{t} 3$ for the first time.

The average age of participants was 10.4 years at $\mathrm{t} 3$ and 12.4 years at $\mathrm{t} 5$. Half of the study sample was male (50\%) and $28.6 \%$ of the children came from immigrant communities. Of the children, $13.3 \%$ had a lower educational background as assessed by their parents, $36.9 \%$ a medium and $49.8 \%$ a high educational background (for further details on the assignment of educational background see [5]).

\subsection{Measures}

\subsubsection{Media Usage}

For wider contextualization, lifetime prevalence of violent media content consumption was queried by the following four questions: "Have you ever played computer games rated 16?", "Have you ever 
played computer games rated 18?", "Have you ever watched films rated 16?" and "Have you ever watched films rated 18?" (two-point scale: no $=0$, yes $=1$ ). All four questions were followed by the supplement "If yes: How often within the last four weeks?" (four-point scale: not at all = 0, 1-2 times = 1, 3-6 times $=2$, more often $=3$ ). The answer "no" to the introductory question was also coded as "not at all $=0$ " for the last four weeks. Separately for computer games and films, violent content consumption games (VC1) and violent content consumption films (VC2) was defined as the highest numerical response on the supplement in the two categories "rated 16" and "rated 18" (SPSS function max.1, range $0-3)$.

\subsubsection{Aggressive Behavior}

Aggressive behavior from the offender's perspective for the school context as well as the context outside school [65-67] was assessed via self-report at $t 3$, $t 4$ and $t 5$, starting from 4 th grade. For the context outside school, students were asked: "Concerning behavior which can happen outside school. Have you ever done one of the following things? (two-point scale: no $=0$, yes $=1$ ). If yes: How often within the last twelve months?" (open response). The following four incidents were queried with regard to aggressive behavior: "Deliberately hurt another child with the result that it cried or was injured", "Threatened another child so that it handed over something to you", "Deliberately damaged windows, telephone booths, street lights or similar things" and "Played with fire or set something on fire". Aggressive behavior perpetration outside school (AB1) was defined as the sum score of these four incidents (range 0-4).

School violence perpetration was queried for the last four weeks preceding the interview by the following items (four-point scale: never, once or twice, three to six times, more often): "Sometimes trouble occurs at school and on the way to school. How often have you done one of the following things within the last four weeks (introduction)"; "I have hit or kicked another student, not for fun", "I have broken some of another student's stuff on purpose", "I have forced another student to do something he/she did not want to do". Aggressive behavior perpetration at school (AB2) was defined as the sum score of the dichotomized (no = 0, yes =1) responses to these three incidents (range 0-3), whereas "never" was recoded to no and "once or twice", "three to six times" and "more often" to yes.

\subsubsection{Empathy}

A German inventory (IVE; adapted from Eyesenck's $\mathrm{I}_{6},[68]$ ) for children at the age of 9-14 was used to assess empathy [69] at t3, t4 and t5. Due to time constraints, a short scale of 4 out of 16 items was constructed, consisting of the following four items (two-point scale: no $=0$, yes $=1$ ): "It troubles me when I see that someone is laughed at" (Em1), "It is hard for me to see someone cry" (Em2), "I often feel compassion for people who are worse off than me" (Em3) and "I feel bad for students who are picked on often" (Em4). High values on this scale stand for pronounced empathy (Cronbachs $\alpha_{t 3}=0.74$, Cronbachs $\alpha_{t 4}=0.79$, Cronbachs $\alpha_{t 5}=0.74$; (for further scale characteristics see [12])). 


\subsection{Procedure}

After obtaining written parental consent, a four-hour paper-pencil interview spread over two consecutive days was completed within the classroom setting on five occasions ( $\mathrm{t} 1$ : November 2005, t2: May 2006, t3: May 2007, t4: May 2008, t5: May 2009). The interviews were conducted by trained interviewers (Bachelor or Master students of psychology). For longitudinal identification, every single student was assigned a unique numeric code which stayed with the school over the study period.

\subsection{Data Analysis}

In the following, $\mathrm{t} 3$ (fourth grade) will be referred to as Time 1, $\mathrm{t} 4$ (fifth grade) as Time 2 and $\mathrm{t} 5$ (sixth grade) as Time 3. For treating missing data (missing values per variable: $4.7 \%-14.5 \%$ ), chained equation modeling [70] was used with the following variables (Time 1, Time 2, Time 3): gender, violent content computer games (VC1), violent content films (VC2), aggressive behavior perpetration outside school (AB1), aggressive behavior perpetration at school (AB2), and empathy (Em1, Em2, Em3, Em4). Predictive mean matching was used for correcting (e.g., only real values were computed). We used the R package "mice” (Multivariate Imputation by Chained Equations; [71]) for this procedure.

To test for changes over time and gender differences, repeated measures (Time 1, Time 2, Time 3) ANOVAs were computed for all included variables using IBM SPSS 19.

The multi-level structure of the data (level 1: measurement time, level 2: individual, level 3: class) was assessed using STATA (Version SE 10). The intra-class-correlation (ICC) was 0.024 at level 3, i.e., at the maximum $2.4 \%$ of the variance of aggressive behavior could be explained by characteristics of the class context in this model (cf. [72,73]). Introducing variables of social and cultural capital (educational background, prosperity, migration background) reduced the conditional ICC to $1.9 \%$ [74]. Considering this small maximum variance being explained at level 3, the multi-level structure was not introduced in structural equation modeling.

To assess the role of empathy as a mediator of the longitudinal relationship between the usage of violent media content and aggressive behavior, trivariate structural equation modeling according to Valkenburg and Peter (cf. [75,76]) was conducted using three measurement times. For all three constructs, latent variables estimated from manifest indicators were used. Weak measurement invariance was tested by constraining the estimate factor loadings to be equal across groups using multi-group factor analyses across time points (group $1=$ Time 1; group $2=$ Time 2; group $3=$ Time 3). As noted by Chen [77], the commonly used chi-square difference test of nested models is almost always significant in large samples and is highly sensitive to departures from multivariate normality. Thus, we used differences in CFI to compare different stages of measurement invariance. A cut-off value of $\triangle \mathrm{CFI}>0.01$ as recommended by Chen [77] for weak invariance was used. Results indicate weak measurement invariance $(\Delta \mathrm{CFI}=0.003)$. In equation modeling, measurement equivalence over time was ensured by restricting the paths of identical manifest variables of Time 1, Time 2 and Time 3 . Furthermore, correlated errors of measurement between identical manifest variables were assumed to account for their inter-correlations over time (Time 1, Time 2, Time 3) [78]. The model was calculated using maximum likelihood estimations. Level of significance was estimated using bootstrap (BC-CI 
95\%) with $K=1,000$ bootstrap samples [79]. Estimations were controlled for study group. Analyses were realized using IBM SPSS Amos 21 [80].

The hypothesized mediation model of empathy, violent content consumption and aggressive behavior is depicted in Figure 1. First, it is assumed that violent content consumption, empathy and aggressive behavior are all three correlated at Time 1. Second, all three constructs have a certain stability over time. Third, violent content consumption at Time 1 is assumed to have a negative influence on empathy at Time 2 (a). Fourth, empathy at Time 2 is predicted to have a negative influence on aggressive behavior at Time 3 (b). Fifth, if empathy completely mediates the significant path from violent content consumption to aggressive behavior (c), the path from violent content consumption at Time 1 on aggressive behavior at Time 3 (c') will be statistically non-significant.

Figure 1. Hypothesized mediation model of empathy, violent content consumption and aggressive behavior.

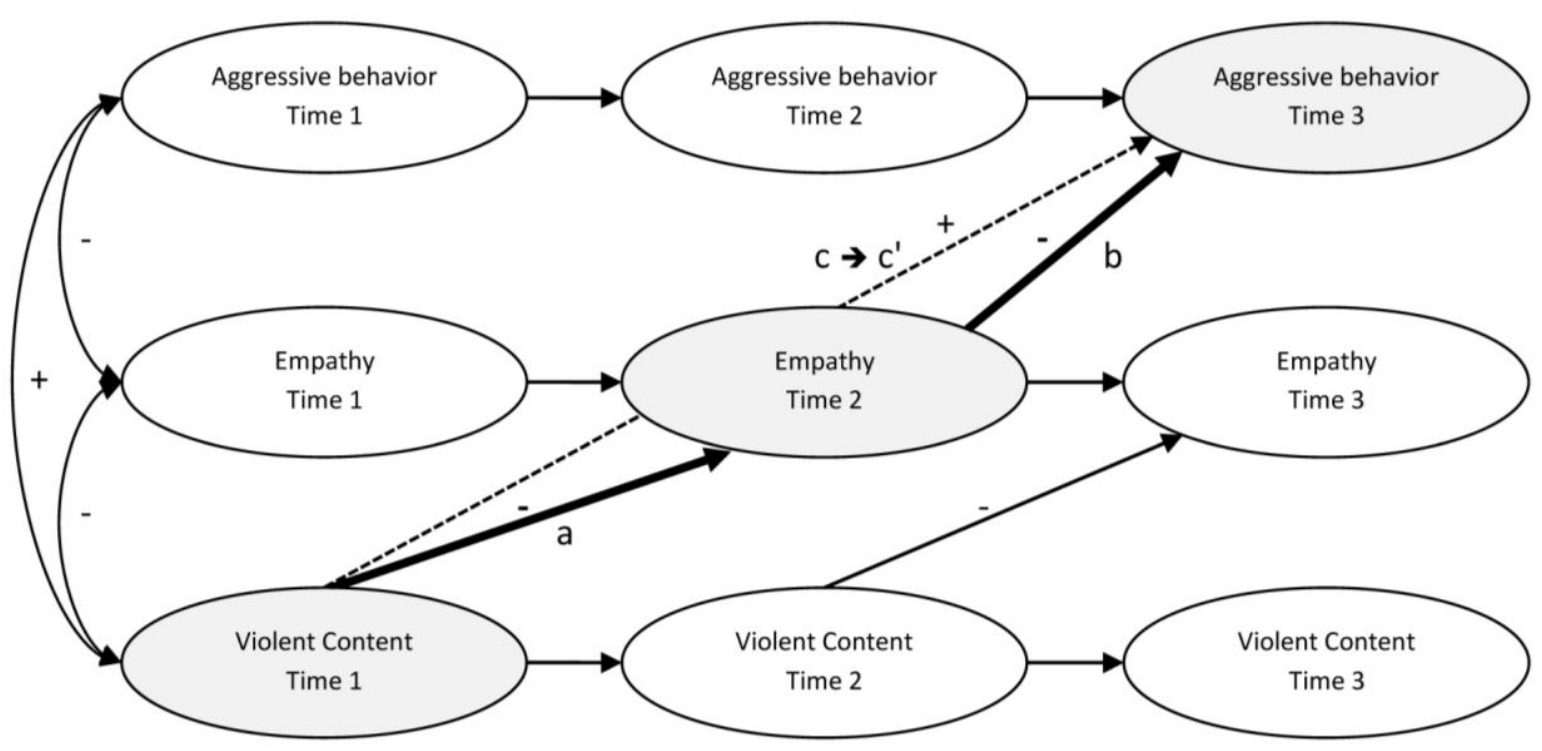

\section{Results}

\subsection{Descriptive Statistics}

Table 1 shows means and standard deviations (total and separated by gender) of all manifest variables for Time 1, Time 2 and Time 3. Regarding violent content, boys had markedly higher mean scores than girls for both video games and films, indicating a higher intensity of violent content consumption. Beyond that, there was, as expected, an overall increase in the consumption of violent content over time. With respect to aggressive behavior, male students were significantly more frequently offenders outside school as well as at school at all three measurement occasions. The overall rate of violence perpetration decreased from Time 1 to Time 3 outside school and increased from Time 1 to Time 3 at school. Both changes were caused only by the reported rates of the boys, whereas girls' rates are rather stable over time. Overall, the mean scores of empathy (Em1-Em4) decreased slightly from Time 1 to Time 3. At all three measurements, boys showed lower scores of empathy. 
Table 1. Descriptive statistics.

\begin{tabular}{|c|c|c|c|c|c|c|c|c|c|c|}
\hline & \multicolumn{9}{|c|}{$M(S D)$} & \multirow{3}{*}{ ANOVA } \\
\hline & \multicolumn{3}{|c|}{ Time 1} & \multicolumn{3}{|c|}{ Time 2} & \multicolumn{3}{|c|}{ Time 3} & \\
\hline & total & boys & girls & total & boys & girls & total & boys & girls & \\
\hline \multicolumn{11}{|l|}{ Media usage } \\
\hline $\begin{array}{l}\text { Violent content VG } \\
\text { (VC1) }\end{array}$ & $\begin{array}{c}0.59 \\
(0.50)\end{array}$ & $\begin{array}{c}0.96 \\
(1.17)\end{array}$ & $\begin{array}{c}0.22 \\
(0.57)\end{array}$ & $\begin{array}{c}0.59 \\
(0.49)\end{array}$ & $\begin{array}{c}0.99 \\
(1.14)\end{array}$ & $\begin{array}{c}0.20 \\
(0.57)\end{array}$ & $\begin{array}{c}0.77 \\
(0.46)\end{array}$ & $\begin{array}{l}1.30 \\
(1.24)\end{array}$ & $\begin{array}{c}0.24 \\
(0.61)\end{array}$ & $\mathrm{t}, \mathrm{g}, \mathrm{t}^{*} \mathrm{~g}$ \\
\hline $\begin{array}{c}\text { Violent content TV } \\
(\mathrm{VC} 2)\end{array}$ & $\begin{array}{c}0.64 \\
(0.94) \\
\end{array}$ & $\begin{array}{r}0.87 \\
(1.06) \\
\end{array}$ & $\begin{array}{c}0.42 \\
(0.74) \\
\end{array}$ & $\begin{array}{c}0.63 \\
(0.89) \\
\end{array}$ & $\begin{array}{r}0.87 \\
(1.03) \\
\end{array}$ & $\begin{array}{r}0.38 \\
(0.67) \\
\end{array}$ & $\begin{array}{c}0.95 \\
(1.02) \\
\end{array}$ & $\begin{array}{r}1.25 \\
(1.11) \\
\end{array}$ & $\begin{array}{r}0.65 \\
(0.82) \\
\end{array}$ & $\mathrm{t}, \mathrm{g}$ \\
\hline \multicolumn{11}{|l|}{ Aggressive behavior } \\
\hline $\begin{array}{l}\text { Perpetration outside } \\
\text { school (AB1) }\end{array}$ & $\begin{array}{c}0.33 \\
(0.70)\end{array}$ & $\begin{array}{c}0.49 \\
(0.86)\end{array}$ & $\begin{array}{l}0.17 \\
(0.45)\end{array}$ & $\begin{array}{c}0.32 \\
(0.68)\end{array}$ & $\begin{array}{c}0.49 \\
(0.82)\end{array}$ & $\begin{array}{l}0.15 \\
(0.44)\end{array}$ & $\begin{array}{l}0.26 \\
(0.64)\end{array}$ & $\begin{array}{l}0.36 \\
(0.78)\end{array}$ & $\begin{array}{c}0.17 \\
(0.45)\end{array}$ & $\mathrm{t}, \mathrm{g}, \mathrm{t} * \mathrm{~g}$ \\
\hline $\begin{array}{l}\text { Perpetration at school } \\
\text { (AB2) }\end{array}$ & $\begin{array}{r}0.19 \\
(0.49) \\
\end{array}$ & $\begin{array}{r}0.29 \\
(0.59) \\
\end{array}$ & $\begin{array}{c}0.09 \\
(0.34)\end{array}$ & $\begin{array}{c}0.15 \\
(0.46) \\
\end{array}$ & $\begin{array}{c}0.23 \\
(0.57) \\
\end{array}$ & $\begin{array}{c}0.07 \\
(0.29) \\
\end{array}$ & $\begin{array}{c}0.27 \\
(0.60) \\
\end{array}$ & $\begin{array}{c}0.41 \\
(0.72) \\
\end{array}$ & $\begin{array}{c}0.12 \\
(0.41) \\
\end{array}$ & $t, g, t * g$ \\
\hline \multicolumn{11}{|l|}{ Empathy } \\
\hline Em1 & $\begin{array}{c}0.77 \\
(0.42)\end{array}$ & $\begin{array}{c}0.69 \\
(0.47)\end{array}$ & $\begin{array}{c}0.85 \\
(0.36)\end{array}$ & $\begin{array}{c}0.77 \\
(0.41)\end{array}$ & $\begin{array}{c}0.70 \\
(0.46)\end{array}$ & $\begin{array}{c}0.87 \\
(0.34)\end{array}$ & $\begin{array}{c}0.75 \\
(0.44)\end{array}$ & $\begin{array}{c}0.63 \\
(0.48)\end{array}$ & $\begin{array}{c}0.87 \\
(0.34)\end{array}$ & $\mathrm{g}$ \\
\hline Em2 & $\begin{array}{c}0.71 \\
(0.45)\end{array}$ & $\begin{array}{c}0.58 \\
(0.50)\end{array}$ & $\begin{array}{c}0.86 \\
(0.37)\end{array}$ & $\begin{array}{c}0.73 \\
(0.45)\end{array}$ & $\begin{array}{c}0.65 \\
(0.48)\end{array}$ & $\begin{array}{c}0.81 \\
(0.39)\end{array}$ & $\begin{array}{c}0.68 \\
(0.47)\end{array}$ & $\begin{array}{c}0.52 \\
(0.50)\end{array}$ & $\begin{array}{c}0.83 \\
(0.38)\end{array}$ & $t, g, t * g$ \\
\hline Em3 & $\begin{array}{c}0.80 \\
(0.40)\end{array}$ & $\begin{array}{c}0.74 \\
(0.44)\end{array}$ & $\begin{array}{c}0.91 \\
(0.34)\end{array}$ & $\begin{array}{c}0.82 \\
(0.39)\end{array}$ & $\begin{array}{c}0.73 \\
(0.44)\end{array}$ & $\begin{array}{c}0.90 \\
(0.30)\end{array}$ & $\begin{array}{c}0.78 \\
(0.42)\end{array}$ & $\begin{array}{c}0.70 \\
(0.46)\end{array}$ & $\begin{array}{c}0.85 \\
(0.36)\end{array}$ & $\mathrm{g}$ \\
\hline Em4 & $\begin{array}{c}0.83 \\
(0.39)\end{array}$ & $\begin{array}{c}0.74 \\
(0.44)\end{array}$ & $\begin{array}{c}0.91 \\
(0.28)\end{array}$ & $\begin{array}{c}0.78 \\
(0.42)\end{array}$ & $\begin{array}{c}0.68 \\
(0.47)\end{array}$ & $\begin{array}{c}0.88 \\
(0.33)\end{array}$ & $\begin{array}{c}0.75 \\
(0.43)\end{array}$ & $\begin{array}{c}0.66 \\
(0.47)\end{array}$ & $\begin{array}{c}0.83 \\
(0.37)\end{array}$ & $\mathrm{t}, \mathrm{g}$ \\
\hline
\end{tabular}

Note: $\mathrm{VG}=$ Video Games. $\mathrm{t}=$ significant main effect time $(\mathrm{t} 3, \mathrm{t} 4, \mathrm{t} 5 ; p<0.05) ; \mathrm{g}=$ significant main effect gender $(p<0.05) ; \mathrm{t}^{*} \mathrm{~g}=$ significant interaction time $(\mathrm{t} 3, \mathrm{t} 4, \mathrm{t} 5)$ by gender $(p<0.05)$.

\subsection{Zero Order Correlations}

The zero order correlation matrix of all manifest variables, which are included in structural equation modeling of Figure 2, can be found split by gender in Table 2, presented for Time 1, Time 2, and Time 3. First, longitudinal correlations between manifest variables of violent content were of medium to large size for male $(0.28 \leq r \leq 0.61)$ and of small to medium size for female $(0.19 \leq r \leq 0.47)$ students; longitudinal correlations between manifest variables of empathy were of small to medium size for male $(0.16 \leq r \leq 0.43)$ and female $\left(0.03^{\text {n.s. }} \leq r \leq 0.35\right)$ students; longitudinal correlations between manifest variables of aggressive behavior were of small to medium size for male $\left(0.04^{\text {n.s. }} \leq r \leq 0.44\right)$ and female $(0.15 \leq r \leq 0.41)$ students as well. Second, for male students, mostly significant, small size, negative cross-sectional correlations $\left(-0.06^{\text {n.s. }} \leq r \leq-0.26\right)$ could be observed between variables of violent content and empathy for Time 1 , Time 2, and Time 3. This was not the case for female students with mostly non-significant associations. Third, for male and female students, there were small negative longitudinal correlations between variables of violent content and empathy (measured at a later point in time; $-0.02^{\text {n.s. }} \leq r \leq-0.19$ ), which were mostly significant for male but not for female students; the same is true for correlations between variables of empathy and violent content at a later point in time (male: $-0.08^{\text {n.s. }} \leq r \leq-0.23$; female: $-0.02^{\text {n.s. }} \leq r \leq-0.15$ ). Fourth, significant small size cross-sectional correlations could be seen between variables of violent content and aggressive behavior for male $(0.16 \leq r \leq 0.28)$ as well as female $\left(0.04^{\text {n.s. }} \leq r \leq 0.26\right)$ students. 
Table 2. Zero order correlations.

\begin{tabular}{|c|c|c|c|c|c|c|c|c|c|c|c|c|c|c|c|c|c|c|c|c|c|c|c|c|}
\hline & \multicolumn{8}{|c|}{ Time 1} & \multicolumn{8}{|c|}{ Time 2} & \multicolumn{8}{|c|}{ Time 3} \\
\hline & $\mathrm{VC} 1$ & $\mathrm{VC} 2$ & Em1 & Em2 & Em3 & Em4 & $\mathrm{AB} 1$ & $\mathrm{AB} 2$ & $\mathrm{VC1}$ & $\mathrm{VC} 2$ & Em1 & Em2 & Em3 & Em4 & $\mathrm{AB} 1$ & AB2 & $\mathrm{VC} 1$ & $\mathrm{VC} 2$ & Em1 & Em2 & Em3 & Em4 & $\mathrm{AB} 1$ & $\mathrm{AB} 2$ \\
\hline \multicolumn{25}{|c|}{ Time 1} \\
\hline $\mathrm{VC1}$ & - & .473 & .020 & .063 & -.085 & .037 & .179 & .202 & .425 & .317 & -.061 & -.077 & -.075 & -.106 & .185 & .193 & .345 & .269 & -.075 & -.120 & .042 & -.111 & .071 & .202 \\
\hline $\mathrm{VC} 2$ & .640 & - & -.038 & -.023 & -.160 & -.075 & .202 & .129 & .260 & .360 & -.099 & -.074 & -.069 & -.180 & .156 & .103 & .189 & .373 & -.110 & -.136 & -.016 & -.060 & .084 & .217 \\
\hline Em1 & -.127 & -.164 & - & .339 & .322 & .324 & -.184 & -.105 & .000 & -.017 & .261 & .158 & .133 & .075 & .044 & .045 & -.007 & -.110 & .193 & .175 & .057 & .138 & -.064 & -.060 \\
\hline Em2 & -.056 & -.065 & .443 & - & .317 & .239 & -.009 & -.036 & .016 & .044 & .234 & .288 & .105 & .141 & .081 & .047 & .021 & .063 & .032 & .312 & .032 & .093 & -.009 & .036 \\
\hline Em3 & -.146 & -.242 & .432 & .431 & - & .303 & -.088 & -.127 & -.105 & -.147 & .155 & .072 & .240 & .100 & .082 & .091 & -.030 & -.062 & .107 & .081 & .152 & .063 & .002 & -.100 \\
\hline Em4 & -.168 & -.158 & .486 & .418 & .421 & - & .050 & -.085 & .005 & .048 & .251 & .143 & .216 & .212 & .086 & .038 & .027 & .032 & .079 & .146 & .143 & .201 & -.037 & -.050 \\
\hline $\mathrm{AB} 1$ & .209 & .164 & -.039 & .003 & -.080 & -.080 & - & .277 & .155 & .157 & -.092 & -.038 & -.099 & -.070 & .269 & .193 & .138 & .130 & -.056 & -.064 & -.036 & -.068 & .153 & .177 \\
\hline $\mathrm{AB} 2$ & .236 & .214 & -.040 & .004 & -.119 & -.130 & .430 & - & .132 & .156 & -.059 & -.049 & -.040 & -.095 & .274 & .270 & .143 & .069 & -.083 & -.156 & -.043 & -.094 & .151 & .370 \\
\hline \multicolumn{25}{|c|}{ Time 2} \\
\hline $\mathrm{VC} 1$ & .540 & .429 & -.127 & -.102 & -.089 & -.156 & .116 & .112 & - & .362 & -.066 & -.102 & -.060 & -.080 & .225 & .189 & .470 & .226 & -.066 & -.143 & -.033 & -.147 & .035 & .254 \\
\hline $\mathrm{VC} 2$ & .481 & .488 & -.152 & -.078 & -.230 & -.181 & .146 & .156 & .605 & - & -.068 & -.033 & -.065 & -.153 & .255 & .140 & .330 & .455 & -.129 & -.117 & .020 & -.100 & .120 & .142 \\
\hline Em1 & -.061 & -.140 & .328 & .322 & .340 & .271 & -.144 & -.042 & -.138 & -.168 & - & .427 & .406 & .478 & -.032 & -.023 & -.006 & -.079 & .327 & .281 & .225 & .264 & .017 & -.043 \\
\hline Em2 & -.023 & -.126 & .249 & .320 & .296 & .257 & -.103 & -.031 & -.139 & -.169 & .446 & - & .274 & .353 & -.054 & -.105 & -.071 & -.049 & .242 & .359 & .148 & .102 & .009 & .026 \\
\hline Em3 & -.103 & -.189 & .239 & .297 & .392 & .392 & -.061 & -.066 & -193 & -.176 & .542 & .529 & - & .293 & .013 & .014 & .013 & -.034 & .245 & .116 & .345 & .267 & -.059 & -.123 \\
\hline Em4 & -.112 & -.188 & .294 & .282 & .337 & .337 & -.167 & -.071 & -193 & -.263 & .596 & .544 & .535 & - & -.045 & -.148 & -.006 & -.099 & .104 & .284 & .200 & .289 & -.013 & -.096 \\
\hline $\mathrm{AB} 1$ & .180 & .241 & -.009 & -.049 & -.135 & -.089 & .377 & .227 & .165 & .244 & -.228 & -.140 & -.237 & -.178 & - & .336 & .185 & .104 & -.032 & -.080 & -.014 & -.152 & .411 & .237 \\
\hline $\mathrm{AB} 2$ & .157 & .195 & -.076 & -.178 & -.235 & -.157 & .180 & .326 & .251 & .186 & -.224 & -.195 & -.214 & -.234 & .345 & - & .160 & .122 & .005 & -.126 & .016 & -.129 & .278 & .258 \\
\hline \multicolumn{25}{|c|}{ Time 3} \\
\hline $\mathrm{VC1}$ & .454 & .276 & -.150 & -.107 & -.151 & -.217 & .185 & .118 & .613 & .545 & -.153 & -.217 & -.231 & -.188 & .208 & .164 & - & .459 & -.127 & -.101 & -.026 & -.044 & .108 & .326 \\
\hline $\mathrm{vC} 2$ & .382 & .430 & -.166 & -.081 & -.065 & -.167 & .135 & .082 & .520 & .481 & -.125 & -.186 & -139 & -.156 & .209 & .150 & .590 & - & -.129 & -.059 & -.048 & -.174 & .183 & .186 \\
\hline Em1 & -.068 & -.103 & .301 & .219 & .187 & .226 & -.089 & .031 & -.178 & -189 & .397 & .391 & .339 & .395 & -.195 & -.114 & -.259 & -.204 & - & .216 & .339 & .373 & -.037 & -.043 \\
\hline Em2 & -088 & -.116 & .169 & .251 & .219 & .156 & -.048 & -.014 & -.063 & -.114 & .362 & .312 & .261 & .328 & -.168 & -.031 & -.186 & -.113 & .447 & - & .350 & .296 & -.097 & -.065 \\
\hline Em3 & -.092 & - -059 & .156 & .175 & .238 & .238 & -.088 & -.069 & -.171 & -.143 & .291 & .292 & .374 & .298 & -134 & - 198 & -.229 & -.199 & .421 & .400 & - & .397 & -.070 & -.065 \\
\hline Em4 & -092 & -.068 & .284 & .301 & .276 & .303 & -.073 & -.036 & -.166 & -.132 & .431 & .309 & .326 & .357 & -.216 & -168 & -.221 & -.121 & .550 & .460 & .442 & - & -.251 & -.049 \\
\hline $\mathrm{AB} 1$ & .084 & .141 & -087 & -.096 & -096 & -088 & .167 & .046 & .119 & .190 & - -183 & -.080 & -.118 & -.116 & .443 & .210 & .202 & .182 & -.153 & -.226 & -.151 & -.251 & - & .207 \\
\hline AB2 & .170 & .134 & -.020 & -.003 & -.044 & -.053 & .230 & .145 & .144 & .179 & -244 & -106 & -120 & -208 & .430 & .280 & .275 & .231 & -.214 & -192 & -.184 & -205 & .411 & - \\
\hline
\end{tabular}

Note: Above the diagonal $=$ female. Below the diagonal $=$ male. VC1 $=$ Violent content consumption computer games. VC2 = Violent content consumption films. Em $1=$ 'It troubles me when I see that someone is laughed at'. Em2 = 'It is hard for me to see someone cry'. Em3 = 'I often feel compassion for people who are worse off than me'. Em4 = 'I feel bad for students who are picked on often'. $\mathrm{AB} 1=$ Violent behavior perpetration outside school. $\mathrm{AB} 2=$ Violent behavior perpetration at school. If not marked differently, all correlations $p<.05$ Grey and italics = non-significant. 
Fifth, longitudinal correlations between variables of violent content and aggressive behavior at a later point in time were of small size for male $\left(0.08^{\text {n.s. }} \leq r \leq 0.24\right)$ and of small to medium size for female $\left(0.07^{\text {n.s. }} \leq r \leq 0.33\right)$ students; longitudinal correlations between aggressive behavior and violent content were of small size for male $\left(0.08^{\text {n.s. }} \leq r \leq 0.21\right)$ as well as female $\left(0.07^{\text {n.s. }} \leq r \leq 0.19\right)$ students. Sixth, for male students, mostly significant, small, negative, cross-sectional correlations $\left(-0.04^{\text {n.s. }} \leq r \leq-0.25\right)$ could be found between variables of empathy and aggressive behavior; for female students these associations were mostly non-significant $\left(-0.01^{\text {n.s. }} \leq r \leq-0.25\right)$. Seventh, for male students, there were mostly non-significant, small, negative longitudinal correlations between variables of empathy and aggressive behavior at a later point in time $\left(-0.01^{\text {n.s. }} \leq r \leq-0.24\right)$. For female students, there was only one significant association. The same is true for associations between variables of aggressive behavior and empathy at a later point in time, with only four significant associations for female students. In sum, the observed associations were stronger for male than for female students with a more coherent picture for males.

\subsection{Longitudinal Mediator Analyses}

The mediation model of Figure 1 (no additional paths included, not controlling for gender) showed an acceptable model fit $\left(\chi^{2}=770.41(252, N=724), C F I=0.91, R M S E A=0.05\right.$ (90\% CI: 0.049 , $0.058), S R M R=0.07)$. The included variables explained $51 \%$ of the variance of violent behavior at Time 3. Due to the observed gender differences in the conducted repeated measures ANOVAs as well as the cross-sectional and longitudinal correlational analyses, we decided to further examine the influence of gender in our mediation model. We first tested for weak measurement invariance using multi-group factor analyses constraining for time and gender (group $1=$ male; group 2 = female); differences in CFI were used to compare the multi-group with the total sample model. Results indicate no measurement invariance for gender $(\triangle \mathrm{CFI}=0.05)$. Due to the apparent gender differences in measurement and the related missing comparability of paths in the multi-group framework, we performed two additional model calculations; separate for male and female students (see Figure 2, all included paths displayed).

The hypothesized mediation model of Figure 1 also showed an acceptable model fit for the male subsample $\left(\chi^{2}=506.97(252, N=362), C F I=0.91, R M S E A=0.05\right.$ (90\% CI: 0.046, 0.060), $S R M R=0.08 ; 44 \%$ explained variance of violent behavior at Time 3). First, violent content and empathy $(\varphi=-0.28$, [BC-CI 95\%: $-0.323,-0.222 ; p<0.01])$, empathy and aggressive behavior $(\varphi=-0.21$, (BC-CI 95\%: $-0.279,-0.126 ; p<0.01))$, as well as violent content and aggressive behavior $(\varphi=0.44$, (BC-CI 95\%: 0.351, 0.527; $p<0.01)$ ) were all significantly correlated at Time 1 . Second, all three constructs showed a certain stability over time. Third, violent content consumption at Time 1 had a significant negative influence on empathy at Time 2 (a), $\beta=-0.08$ (BC-CI 95\%: $-0.125,-0.026 ; p<0.01$ ). Fourth, empathy at Time 2 had a significant influence on aggressive behavior at Time 3 (b), $\beta=-0.14$ [BC-CI 95\%: $-0.200,-0.077 ; p<0.01$ ]. Fifth, the path from violent content consumption at Time 1 on aggressive behavior at Time 3 (c') was statistically non-significant $(\beta=0.01$, (BC-CI 95\%: $-0.054,0.065 ; p=0.84)$ ). C was $\beta=0.25$ (BC-CI 95\%: 0.182 , $0.360 ; p<0.01$ ) in a model without any additional explanatory variables (BC-CI 95\% with 
$K=1,000$ bootstrap samples; $\chi^{2}=4.382(3, N=362), C F I=0.99, R M S E A=0.04$ (90\% CI: 0.000, $0.102), S R M R=0.01)$, thus indicating a complete mediation by empathy.

Figure 2. Observed mediation model of empathy, violent content consumption and aggressive behavior by gender (male/female).

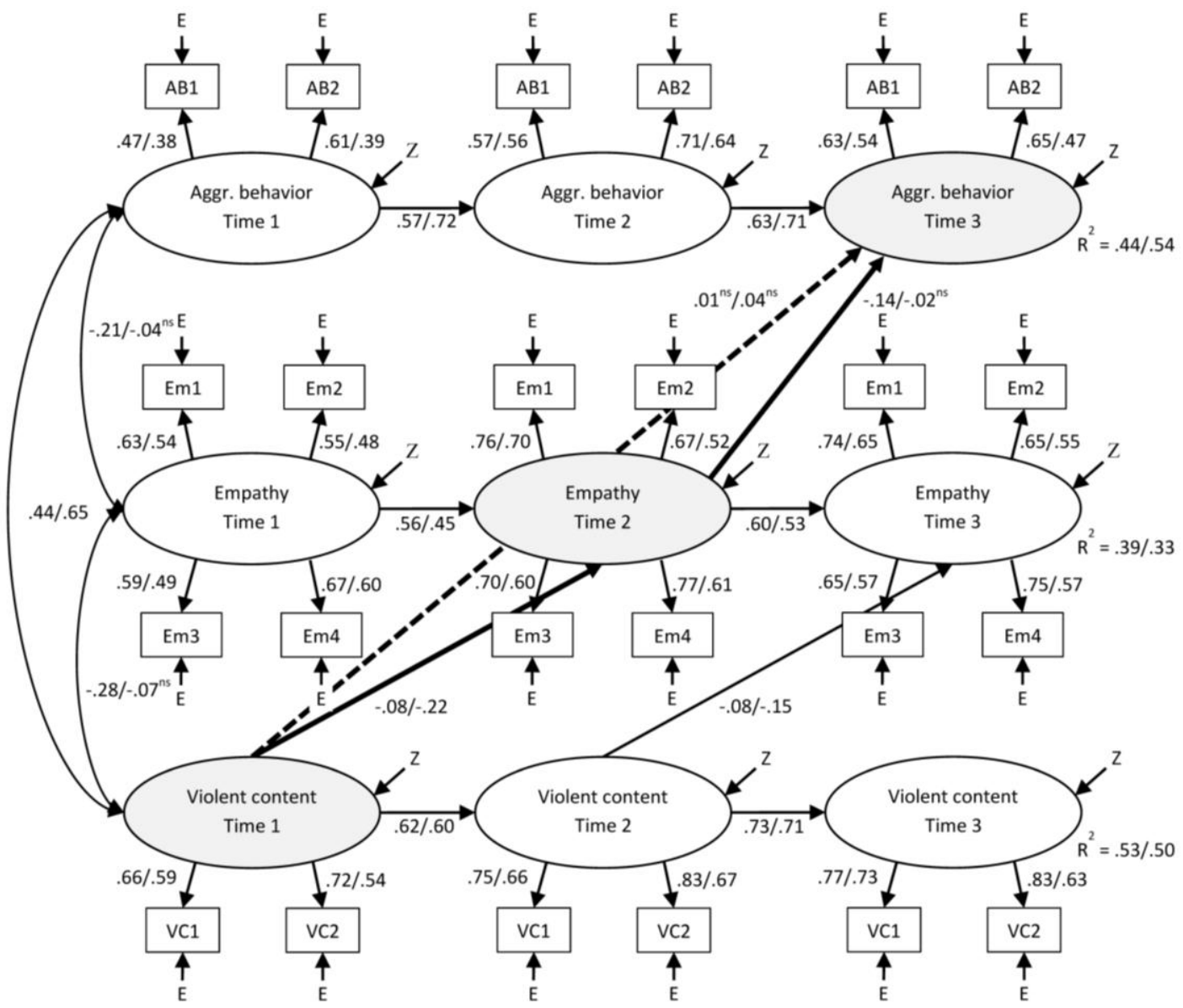

Note: Male/female. All standardized parameters. If not marked differently, all paths at least $p<0.01$.

The model for the female subsample showed an overall comparable model fit $\left(\chi^{2}=475.61\right.$ ( 252 , $N=362), C F I=0.88, R M S E A=0.05$ (90\% CI: 0.043, 0.056), SRMR $=0.06 ; 54 \%$ explained variance of violent behavior at Time 3), but a pattern of results departing from the hypothesized mediation model of Figure 1. First, only violent content and aggressive behavior ( $\varphi=0.65$, (BC-CI 95\%: 0.526, $0.778 ; p<0.01))$ were significantly correlated at Time 1 ; violent content and empathy $(\varphi=-0.07$, (BC-CI 95\%: $-0.161,0.010 ; p=0.08)$ ), empathy and aggressive behavior $(\varphi=-0.04$, (BC-CI 95\%: $-0.181,0.032 ; p=0.29)$ ). Second, in line with the hypothesized mediation model, all three constructs showed certain stability over time; with lower stabilities for empathy and higher stabilities for aggressive behavior compared to the male subsample. Third, violent content consumption at Time 1 had again a significant negative influence on empathy at Time 2 (a), $\beta=-0.22$ (BC-CI 95\%: -0.269 , $-0.175 ; p<0.01)$. Fourth, differing from our assumptions, empathy at Time 2 had no significant influence on aggressive behavior at Time 3 (b), $\beta=-0.02$ (BC-CI 95\%: $-0.086,0.030 ; p=0.35$ ). Fifth, the path from violent content consumption at Time 1 on aggressive behavior at Time 3 was again statistically non-significant (c'), $\beta=0.04$, (BC-CI 95\%: $-0.137,0.223 ; p=0.64$ ). Hence, for the female subsample no mediation by empathy of the longitudinal relationship between violent media 
content consumption and aggressive behavior can be observed (Significant path c ( $\beta=0.42$ (BC-CI 95\%: 0.246, 0.605; $p<0.01)$ ); model fit (BC-CI 95\% with $K=1,000$ bootstrap samples): $\chi^{2}=0.80$ $(3, N=362), C F I=1.0 ., R M S E A=0.00$ (90\% CI: 0.000, 0.049), SRMR =0.01).

\section{Discussion}

\subsection{Summary of Findings and Theoretical Implications}

The aim of this study was to thoroughly investigate the link between violent media consumption and aggressive behavior. Using a large longitudinal student sample, the role of empathy as a possible mediator of this relationship was of special interest. For the recorded variables of aggressive behavior, slightly higher prevalence rates compared to a representative nationwide school survey [67] using a similar questionnaire for assessing aggressive behavior could be observed for the Berlin Longitudinal Study Media - as was to be expected for a metropolis (act of violence: $23 \%$ vs. 15\%; damage to property: $9 \%$ vs. $7 \%$; theft: $7 \%$ vs. $4 \%$ ). Similar gender differences in aggressive behavior to those reported in the nationwide survey [67] were found in the present data. The observed higher rate of violence perpetration of boys (cf. [18,27]), the observed higher preference of boys for violent media content (cf. [5,28]) as well as the observed lower empathic scores of boys (cf. [28,62]) have been repeatedly documented.

Bivariate cross-sectional and longitudinal correlational analyses showed a result pattern comparable to previous studies, with significant associations between higher violent media content consumption and lower empathy (cf. [23,28]), between lower empathy and a higher rate of aggressive behavior (cf. $[23,24]$ ), and between higher violent content consumption and a higher rate of aggressive behavior (cf. $[17,18]$ ). Effect sizes were shown to lie within the range of meta-analytic results reported in the introduction [7-9]. Concerning gender differences, our analyses produced mixed results: They are in line with meta-analytic evidence (cf. $[7,8,81]$ ) for the link between violent content consumption and aggressive behavior, revealing no gender differences. They dissent from meta-analytic evidence (cf. $[7,8,81]$ ), however, with regard to the associations of violent content and empathy as well as empathy and aggression, which are less pronounced and often non-significant for the female subsample.

The conducted trivariate structural equation modeling, assessing the role of empathy as a mediator of the observed longitudinal relationship between violent media content consumption and aggressive behavior, showed a full mediation of this relationship by empathy for the male, but not the female subsample. To our knowledge, this is the first study to demonstrate this mediation effect with longitudinal data using three measurements (cf. [23,28]). There are several possible explanations for gender differences in the mediation effect: First, female students showed overall lower scores in aggressive behavior and violent content consumption as well as higher scores in empathy, possibly indicating either a threshold effect of empathy or a varying proneness to (mediating) media effects. Second, those few female students in the sample with aggressive behavior at Time 1 were more stable in their behavior over time (cf. [82,83]); for male students more variation could be observed. Thus aggressive behavior at Time 3 can be explained by earlier aggressive behavior to a larger extent for female than for male students. Although having found no mediation effect for the female subsample, structural equation modeling still produced significant results: First, violent content consumption and a 
higher rate of aggressive behavior were highly correlated at Time 1 (and even more marked for females than for males, $z=4.06$ ). Second, violent media content consumption was causally related to lower empathy.

One explanatory framework for the mediating effect of empathy is given by the long-term components of the $G A M$ by Anderson and colleagues $[10,11,50]$ and the independent explanatory pathway of habituation or desensitization to media violence: repeated reception phases of violent media content over a longer period of time lead to aggressive beliefs and attitudes, aggressive perceptual schemata, a hostile attribution bias, aggressive behavior scripts, and a desensitization to violence via processes of learning, rehearsal and reinforcement of aggression-related knowledge structures. A second explanatory framework is given by the downward spiral model of Slater and colleagues [17,48]. This explains the relationship between the consumption of violent media content and aggressive behavior as an interaction between predispositions and violent media content usage; both are not mutually exclusive and can jointly have negative, antisocial and destructive consequences. Empathy might be one of these key mediating predispositions.

Results of additional analyses not reported here (cf. [12,84]) also showed lower scores of empathy to be associated with higher media usage times [85]. For explaining this relationship, a hypothesis of time displacement could be assumed [63,84]: Children who spend many hours a day in digital worlds, only interacting there with media characters or other user's avatars, seem to have an underdeveloped ability of empathy independently of the chosen content. Para-social relationships with computer characters and avatars would thus appear an ineffective substitute for social interactions in the real world, which form the basis of developing empathic skills-insofar as they displace human relationships, face-to-face communication and interaction to a great extent. Taken together, empathic abilities seem to unfold a key mediating role between a problematic media usage, with regard to content and time, and variables of social coexistence.

We do not want to go as far as Carnagey and colleagues [60] and fear the danger of an increasing, unnoticeable overall desensitization to violence through (as just shown, not only) violent media content consumption. There are too many personal and environmental variables of equivalent or greater explanatory power involved. This is also true for empathy, and all these variables interact with each other [12]. Yet, applying the GAM by Anderson and colleagues [10,11,50], desensitization is just one of five possible pathways from violent media content consumption to increased aggressive behavior. If one also considers related changes in values, norms and attitudes, however, deeper reflection is needed. Last but not least, violent media content consumption is the variable which could be most easily targeted and influenced as compared to many others. Formulated as a question: Is violent media content consumption necessarily part of an overall deviant lifestyle [86], thus acting as a reinforcer of related values, norms and attitudes?

\subsection{Limitations and Future Research}

In spite of having identified a causal relationship between violent content consumption and empathy and having identified the role of empathy as a mediator of the relationship between violent media consumption and aggressive behavior for the male subsample, there are certain limitations to the presented results (cf. [87]). First, evidence is restricted to those variables that have been included in the 
study and the analysis. Other variables of possibly equivalent or higher predictive power, e.g., trait aggression, for which there is longitudinal evidence of moderating the relationship between violent content consumption and physical aggression [28], might alter the presented results. Second, having drawn a representative sample of primary grade school children in the state of Berlin, the presented results are strictly speaking representative only of Berlin children in this age group. Third, having assessed all variables of interest via self-report, some results might differ if other instruments or procedures, such as peer-nomination, had been used instead. Nevertheless, having these limitations in mind, the present study added further longitudinal evidence for the influence of violent media consumption on aggressive behavior, which in this study is fully mediated by empathy for the male subsample. By this means, the significance of empathy was shown. In future studies, the observed relationships and gender differences need to be validated, also with different populations and older age groups. Furthermore, this should be realized more frequently in longitudinal studies using more than two times of measurement.

\section{Conclusions}

The questions of how and under what premises media usage can promote aggressive behavior remains a complex as well as controversially discussed issue in research. This is partially due to the fact that most possible risk factors of aggression interact with a specific media usage behavior and possibly moderate or mediate the negative effect of violent media consumption on aggression. This study was the first to examine the role of empathy as a mediator of the relationship between violent media content usage and aggressive behavior using three measurement times. Empathy was shown to fully mediate this relationship in the male subsample. In future studies, emphasis should be placed on conducting longitudinal studies with at least three times of measurement to ensure that possible mediating pathways can be revealed.

\section{Acknowledgments}

The study was financed by the Volkswagen Foundation. No conditions whatsoever were imposed on the financing. We thank the 1207 Berlin school children and their 1085 parents who participated in the study and the 47 junior school headmasters and 89 teachers for helping us to carry out the study at their schools and for providing class time.

\section{Author Contributions}

Conceived, designed and conducted the study: Thomas Mößle and Florian Rehbein. Analyzed the data: Thomas Mößle and Sören Kliem. Wrote the manuscript: Thomas Mößle. Commented on the manuscript: Florian Rehbein and Sören Kliem. Provided intellectual input: Florian Rehbein and Sören Kliem.

\section{Conflicts of Interest}

The authors declare no conflict of interest. 


\section{References}

1. Wilson, B.J.; Smith, S.L.; Potter, W.J.; Kunkel, D.; Linz, D.; Colvin, C.M.; Donnerstein, E. Violence in children's television programming: Assessing the risks. J. Commun. 2002, 52, 5-35.

2. Smith, S.L.; Lachlan, K.; Tamborini, R. Popular video games: Quantifying the presentation of violence and its context. J. Broadcast. Electron. Media 2003, 47, 58-76.

3. Thompson, K.M.; Haninger, K. Violence in e-rated video games. J. Am. Med. Assoc. 2001, 268, 591-598.

4. Feierabend, S.; Karg, U.; Rathgeb, T. JIM-Studie 2012. Jugend, Information, (Multi-)media. Basisuntersuchung zum Medienumgang 12- bis 19-jähriger. [Jim-Study 2012. Youth, Information, (Multi-)Media. Basic Study on the Media Behavior of 12-to 19-Year-Olds]; Medienpädagogischer Forschungsverbund Südwest: Stuttgart, Germany, 2012.

5. Mößle, T.; Kleimann, M.; Rehbein, F.; Pfeiffer, C. Media use and school achievement—Boys at risk? Br. J. Dev. Psychol. 2010, 28, 699-725.

6. Feierabend, S.; Karg, U.; Rathgeb, T. KIM-Studie 2012. Kinder + Medien, Computer + Internet. Basisuntersuchung zum Medienumgang 6- bis 13-jähriger in Deutschland. [KIM-Study 2012. Children + Media, Computer + Internet. Basic Study on the Media Behaviour of 6- to 13-Year-Olds in Germany]; Medienpädagogischer Forschungsverbund Südwest: Stuttgart, Germany, 2013.

7. Anderson, C.A.; Ihori, N.; Bushman, B.J.; Rothstein, H.R.; Shibuya, A.; Swing, E.L.; Saleem, M. Violent video game effects on aggression, empathy, and prosocial behavior in eastern and western countries: A meta-analytic review. Psychol. Bull. 2010, 136, 151-173.

8. Paik, H.; Comstock, G. The effects of television violence on antisocial behavior: A meta-analysis. Commun. Res. 1994, 21, 516-546.

9. Sherry, J.L. The effects of violent video games on aggression: A meta-analysis. Hum. Commun. Res. 2001, 27, 409-431.

10. Anderson, C.A.; Bushman, B.J. Effects of violent video games on aggressive behavior, aggressive cognition, aggressive affect, physiological arousal, and prosocial behavior: A meta-analytic review of the scientific literature. Psychol. Sci. 2001, 12, 353-359.

11. Anderson, C.A.; Gentile, D.A.; Buckley, K.E. Violent Video Game Effects on Children and Adolescents; Oxford University Press: New York, NY, USA, 2007.

12. Mößle, T. Dick, dumm, abhängig, gewalttätig? Problematische Mediennutzungsmuster und ihre Folgen im Kindesalter. Ergebnisse des Berliner Längsschnitt Medien. ["Fat, Stupid, Addicted, Violent?" Problematic Media Usage Behavior and Its Consequences in Childhood. Results of the Berlin Longitudinal Study Media]; Nomos Verlag: Baden Baden, Germany, 2012.

13. Ferguson, C.J. Evidence for publication bias in video game violence effects literature: A meta-analytic review. Aggress. Violent Behav. 2007, 12, 470-482.

14. Ferguson, C.J. The good, the bad and the ugly: A meta-analytic review of positive and negative effects of violent video games. Psychiatr. Q. 2007, 78, 309-316.

15. Ferguson, C.J.; Kilburn, J. The public health risks of media violence: A meta-analytic review. J. Pediatr. 2009, 154, 759-763. 
16. Ferguson, C.J.; Kilburn, J. Much ado about nothing: The misestimation and overinterpretation of violent video game effects in eastern and western nations: Comment on anderson et al. Psychol. Bull. 2010, 136, 174-178.

17. Slater, M.D.; Henry, K.L.; Swaim, R.C.; Cardador, J.M. Vulnerable teens, vulnerable times. Commun. Res. 2004, 31, 642-668.

18. Ribeaud, D.; Eisner, M. Risk factors for aggression in pre-adolescence: Risk domains, cumulative risk and gender differences-Results from a prospective longitudinal study in a multi-ethnic urban sample. Eur. J. Criminol. 2010, 7, 460-498.

19. Warr, M. Companions in Crime: The Social Aspects of Criminal Conduct; Cambridge University Press: New York, NY, USA, 2002.

20. Keenan, K.; Loeber, R.; Zhang, Q.; Stouthamer-Loeber, M.; van Kammen, W.B. The influence of deviant peers on the development of boys' disruptive and delinquent behavior: A temporal analysis. Dev. Psychopathol. 1995, 7, 715-726.

21. Anderson, C.A.; Bushman, B.J. Human aggression. Annu. Rev. Psychol. 2002, 53, $27-51$.

22. Huesmann, L.R.; Guerra, N.G. Children's normative beliefs about aggression and aggressive behavior. J. Personal. Soc. Psychol. 1997, 72, 408-419.

23. Bartholow, B.D.; Sestir, M.A.; Davis, E.B. Correlates and consequences of exposure to video game violence: Hostile personality, empathy, and aggressive behavior. Personal. Soc. Psychol. Bull. 2005, 31, 1573-1586.

24. Roberts, W.; Strayer, J. Empathy, emotional expressiveness, and prosocial behavior. Child Dev. 1996, 67, 449-470.

25. Loeber, R.; Farrington, D.P. Young children who commit crime: Epidemiology, developmental origins, risk factors, early interventions, and policy implications. Dev. Psychopathol. 2000, 12, 737-762.

26. White, J.L.; Moffitt, T.E.; Caspi, A.; Bartusch, D.J.; Needles, D.J.; Stouthammer-Loeber, M. Measuring impulsivity and examining its relationship to delinquency. J. Abnorm. Psychol. 1994, 103, 192-205.

27. Archer, J. Sex differences in aggression in real-world settings: A meta-analytic review. Rev. Gen. Psychol. 2004, 8, 291-322.

28. Krahé, B.; Möller, I. Longitudinal effects of media violence on aggression and empathy among german adolescents. J. Appl. Dev. Psychol. 2010, 31, 401-409.

29. Weiler, S. Die neue Mediengeneration. Medienbiographien als medienpädagogische Prognoseinstrumente. Eine empirische Studie über die Entwicklung von Medienpräferenzen. [The New Media Generation. Media Biographies as Media-Pedagogical Forecasting Tool. An Empirical Study on the Development of Media Preferences]; Reinhard Fischer: München, Germany, 1999.

30. Przybylski, A.K.; Ryan, R.M.; Rigby, C.S. The motivating role of violence in video games. Personal. Soc. Psychol. Bull. 2009, 35, 243-259.

31. Robinson, T.N.; Wilde, M.L.; Navracruz, L.C.; Haydel, K.F.; Varady, A. Effects of reducing children's television and video game use on aggressive behavior: A randomized controlled trial. Arch. Pediatr. Adolesc. Med. 2001, 155, 17-23. 
32. Von Salisch, M.; Kristen, A.; Oppl, C. Computerspiele mit und ohne Gewalt. Auswahl und Wirkung bei kindern. [Computer Games with and without Violence. Selection by Children and Effects on Children]; Kohlhammer: Stuttgart, Germany, 2007.

33. Ferguson, C.J. Video games and youth violence: A prospective analysis in adolescents. J. Youth Adolesc. 2011, 40, 377-391.

34. Janssen, I.; Boyce, W.F.; Pickett, W. Screen time and physical violence in 10 to 16-year-old canadian youth. Int. J. Public Health 2012, 57, 325-331.

35. Morris, R.G.; Johnson, M.C. Sedentary activities, peer behavior, and delinquency among american youth. Crime Delinquency 2010, doi:10.1177/0011128710386205.

36. Von Salisch, M.; Vogelgesang, J.; Kristen, A.; Oppl, C. Preference for violent electronic games and aggressive behavior among children: The beginning of the downward spiral? Media Psychol. 2011, 14, 233-258.

37. Gentile, D.A.; Gentile, J.R. Violent video games as exemplary teachers: A conceptual analysis. J. Youth Adolesc. 2008, 37, 127-141.

38. Hopf, W.H.; Huber, G.L.; Weiß, R.H. Media violence and youth violence- a 2-year longitudinal study. J. Media Psychol.: Theor. Methods Appl. 2008, 20, 79-96.

39. Wallenius, M.; Punamäki, R.-L. Digital game violence and direct aggression in adolescence: A longitudinal study of the roles of sex, age, and parent-child communication. J. Appl. Dev. Psychol. 2008, 29, 286-294.

40. Möller, I.; Krahé, B. Exposure to violent video games and aggression in german adolescents: A longitudinal analysis. Aggress. Behav. 2009, 35, 75-89.

41. Hofferth, S.L. Home media and children's achievement and behavior. Child Dev. 2010, 81, 1598-1619.

42. Zimmerman, F.J.; Glew, G.M.; Christakis, D.A.; Katon, W. Early cognitive stimulation, emotional support, and television watching as predictors of subsequent bullying among grade-school children. Arch. Pediatr. Adolesc. Med. 2005, 159, 384-388.

43. Christakis, D.A.; Zimmerman, F.J. Violent television viewing during preschool is associated with antisocial behavior during school age. Pediatrics 2007, 120, 993-999.

44. Anderson, D.R.; Huston, A.C.; Schmitt, K.L.; Linebarger, D.L.; Wright, J.C. Early childhood television viewing and adolescent behavior. Monogr. Soc. Res. Child Dev. 2001, 66, 1-143.

45. Chowhan, J.; Stewart, J.M. Television and the behaviour of adolescents: Does socio-economic status moderate the link? Soc. Sci. Med. 2007, 65, 1324-1336.

46. Ostrov, J.; Gentile, D.A.; Crick, N.R. Media exposure, aggression and prosocial behavior during early childhood: A longitudinal study. Soc. Dev. 2006, 15, 612-627.

47. Anderson, C.A.; Sakamoto, A.; Gentile, D.A.; Ihori, N.; Shibuya, A.; Yukawa, S.; Naito, M.; Kobayashi, K. Longitudinal effects of violent video games on aggression in japan and the united states. Pediatrics 2008, 122, e1067-e1072.

48. Slater, M.D.; Henry, K.L.; Swaim, R.C.; Anderson, L.L. Violent media content and aggressiveness in adolescents: A downward spiral model. Commun. Res. 2003, 30, 713-736.

49. Johnson, J.G.; Cohen, P.; Smailes, E.M.; Kasen, S.; Brook, J.S. Television viewing and aggressive behavior during adolescence and adulthood. Science 2002, 295, 2468-2471. 
50. Anderson, C.A.; Dill, K.E. Video games and aggressive thoughts, feelings, and behavior in the laboratory and in life. J. Personal. Soc. Psychol. 2000, 78, 772-790.

51. Berkowitz, L. Aggression: Its Causes, Consequences, and Control; McGraw Hill: New York, NY, USA, 1993.

52. Bandura, A. Aggression: A Social Learning Analysis; Prentice-Hall: Englewood Cliffs, NJ, USA, 1973.

53. Crick, N.R.; Dodge, K.A. A review and reformulation of social information-processing mechanisms in children's social adjustment. Psychol. Bull. 1994, 115, 74-101.

54. Geen, R.G. Human Aggression; McGraw Hill: Pacific Grove, CA, USA, 1990.

55. Huesmann, L.R. Psychological processes promoting the relation between exposure to media violence and aggressive behavior by the viewer. J. Soc. Issues 1986, 42, 125-140.

56. Zillmann, D. Cognition-excitation interdependencies in aggressive behavior. Aggress. Behav. 1983, 14, 51-64.

57. Möller, I. Mediengewalt und Aggression: Eine längsschnittliche Betrachtung des Zusammenhangs am Beispiel des Konsums gewalthaltiger Bildschirmspiele. [Media Violence and Aggression: A Longitudinal Examination of This Relationship Using the Example of Violent Computer Game Usage]; Universität Potsdam: Potsdam, Germany, 2006.

58. Mößle, T.; Kleimann, M.; Rehbein, F. Bildschirmmedien im Alltag von Kindern und Jugendlichen: Problematische Mediennutzungsmuster und ihr Zusammenhang mit Schulleistungen und Aggressivität. [Screen Media in Everyday Life of Children and Adolescents: Problematic Usage Patterns and Their Relation to School Success and Aggressive Behavior]; Nomos: Baden-Baden, Germany, 2007.

59. Bartholow, B.D.; Bushman, B.J.; Sestir, M.A. Chronic violent video game exposure and desensitization to violence: Behavioral and event-related brain potential data. J. Exp. Soc. Psychol. 2006, 42, 532-539.

60. Carnagey, N.L.; Anderson, C.A.; Bushman, B.J. The effect of video game violence on psychological desensitization to real-life violence. J. Exp. Soc. Psychol. 2007, 43, 489-496.

61. Krahé, B.; Möller, I.; Huesmann, L.R.; Kirwil, L.; Felber, J.; Berger, A. Desensitization to media violence: Links with habitual media violence exposure, aggressive cognitions, and aggressive behavior. J. Personal. Soc. Psychol. 2011, 100, 630-646.

62. Funk, J.B.; Baldacci, H.B.; Pasold, T.; Baumgardner, J. Violence exposure in real-life, video games, television, movies, and the internet: Is there desensitization? J. Adolesc. 2004, 27, $23-40$.

63. Kleimann, M.; Mößle, T. The logs of eliza and other media stories. Behavioral and developmental effects of a school based media education program-Berlin longitudinal study media. Int. J. Behavi. Dev. 2008, 32, 5-9.

64. Kleimann, M. Medienlotsen gesucht! Konzeption und Evaluation einer Unterrichtseinheit zur Prävention problematischer Mediennutzungsmuster bei Schülerinnen und Schülern dritter bis fünfter Klassen im Rahmen des Berliner Längsschnitt Medien. [Media Pilots Wanted! Conception and Evaluation of School-Based Lessons for the Prevention of Problematic Media Usage of Third to Fifth Graders in the Context of the Berlin Longitudinal Study Media]; Nomos: Baden Baden, Germany, 2011. 
65. Wilmers, N.; Enzmann, D.; Schaefer, D.; Herbers, K.; Greve, W.; Wetzels, P. Jugendliche in Deutschland zur Jahrtausendwende: Gefährlich oder gefährdet? [Youths in Germany at the Turn of the Millenium: Dangerous or Endangered?]; Nomos: Baden-Baden, Germany, 2002.

66. Lösel, F. Handlungskontrolle und Jugenddelinquenz. [Agency and Youth Delinquency]; Enke: Stuttgart, Germany, 1975.

67. Baier, D.; Pfeiffer, C.; Rabold, S.; Simonson, J.; Kappes, C. Kinder und Jugendliche in Deutschland: Gewalterfahrungen, Integration, Medienkonsum. Zweiter Bericht zum gemeinsamen Forschungsprojekt des Bundesministerium des Innern und des KFN. [Children and Adolescents in Germany: Experience of Violence, Integration, Media Consumption]; Forschungsbericht Nr. 109; Kriminologisches Forschungsinstitut Niedersachsen: Hannover, Germany, 2010.

68. Eysenck, S.B.G.; Eysenck, H.J. Impulsiveness and venturesomeness in children. Personal. Individ. Differ. 1980, 1, 73-78.

69. Stadler, C.; Janke, W.; Schmeck, K. IVE: Inventar zur Erfassung von Impulsivität, Risikoverhalten und Empathie bei 9- bis 14-jährigen Kindern. [IVE: Inventory for the Assessment of Impulsivity, Risk Behavior and Empathy for 9- to 14-Year-Old Children]; Hogrefe: Göttingen, Germany, 2004.

70. White, I.R.; Royston, P.; Wood, A.M. Multiple imputation using chained equations: Issues and guidance for practice. Stat. Med. 2011, 30, 377-399.

71. Van Buuren, S.; Groothuis-Oudshoorn, K. Mice: Multivariate imputation by chained equations in R. J. Stat. Softw. 2011, 45, 1-67.

72. Hox, J. Multilevel Analysis: Techniques and Applications; Lawrence Erlbaum Associates: Mahwah, NJ, USA, 2002.

73. Snijders, T.A.B.; Bosker, R.J. Multilevel Analysis. An Introduction to Basic and Advanced Multilevel Modelling; Sage Publications: London, UK, 1999.

74. Oberwittler, D. Geschlecht, Ethnizität und sozialräumliche Benachteiligung. [Gender, ethnicity and socio-environmental disadvantage]. In Geschlecht-Gewalt-Gesellschaft (Otto-von-Freising-Tagungen der katholischen Universität Eichstätt-Ingolstadt); Lamnek, S., Boatca, M., Eds.; Leske und Budrich: Opladen, Germany, 2003; Volume 4, pp. 269-294.

75. Valkenburg, P.M.; Peter, J. The effects of instant messaging on the quality of adolescents' existing friendships: A longitudinal study. J. Commun. 2009, 59, 79-97.

76. Valkenburg, P.M.; Peter, J. The differential susceptibility to media effects model. J. Commun. 2013, 63, 221-243.

77. Chen, F.F. Sensitivity of goodness of fit indexes to lack of measurement invariance. Struct. Equ. Model. 2007, 14, 464-504.

78. Van Aken, M.A.; Helmke, A.; Schneider, W. Selbstkonzept und Leistung-Dynamik ihres Zusammenspiels: Ergebnisse aus dem Scholastik-Projekt. [Self concept and achievement-dynamics of their interplay: Results from the scholastik-project]. In Entwicklung im Grundschulalter; Weinert, F.E., Helmke, A., Eds.; Psychologie Verlags Union: Weinheim, Germany, 1997; pp. 341-350.

79. Preacher, K.J.; Hayes, A.F. Asymptotic and resampling strategies for assessing and comparing indirect effects in multiple mediator models. Behav. Res. Methods 2008, 40, 879-891.

80. Arbuckle, J.L. Amos, 7.0; SPSS: Chicago, IL, USA, 2006. 
81. Anderson, C.A.; Berkowitz, L.; Donnerstein, E.; Huesmann, L.R.; Johnson, J.D.; Linz, D.; Malamuth, N.M.; Wartella, E. The influence of media violence on youth. Psychol. Sci. Public Interest 2003, 4, 81-110.

82. Huizinga, D.; Miller, S.; Conduct Problems Prevention Research Group. Developmental Sequences of Girls Delinquent Behavior; U.S. Department of Justice, Office of Justice Programs, Office of Juvenile Justice and Delinquency Prevention: Washington, DC, USA, 2013.

83. Harachi, T.W.; Fleming, C.B.; White, H.R.; Ensminger, M.E.; Abbott, R.D.; Catalano, R.F.; Haggerty, K.P. Aggressive behavior among girls and boys during middle childhood: Predictors and sequelae of trajectory group membership. Aggress. Behav. 2006, 32, 279-293.

84. Mößle, T.; Roth, C. Gewaltmediennutzung und Gewaltdelinquenz im Grundschulalter. Ergebnisse einer Längsschnittstudie. [Violent Media Usage and Violent Behavior in Elementary School. Results of a Longitudinal Study]; Medienheft: Zürich, Germany, 2009.

85. Bajovic, M. Violent video gaming and moral reasoning in adolescents: Is there an association? Educ. Media Int. 2013, 50, 177-191.

86. Lösel, F.; Bliesener, T.; Bender, D. Social information processing, experiences of aggression in social contexts, and aggressive behavior in adolescents. Crim. Justice Behav. 2007, 34, 330-347.

87. Mößle, T.; Rehbein, F. Predictors of problematic video game usage in childhood and adolescence. Sucht 2013, 59, 129-142.

(C) 2014 by the authors; licensee MDPI, Basel, Switzerland. This article is an open access article distributed under the terms and conditions of the Creative Commons Attribution license (http://creativecommons.org/licenses/by/3.0/). 